Für den vorliegenden Journal Club habe ich drei Studien ausgewählt, die ich für unbedingt beachtenswert halte. Little et al. zeigen, dass ein bislang wenig verbreitetes körpertherapeutisches Verfahren, die Alexander-Technik, Rückenschmerzen auch langfristig überdurchschnittlich gut reduzieren kann. Walach et al. untersuchen die Wirkung von Fernheilung auf das Chronic Fatigue Syndrome und finden einmal mehr Hinweise auf die Bedeutung von Patientenerwartungen für das Therapieergebnis. Dusek et al. vom BensonHenry Institute für Mind Body Medicine, Massachusetts, belegen die Auswirkungen von Entspannungsverfahren auf intrazelluläre Mechanismen der Genexpression, welche die klinischen Langzeitwirkungen der Relaxation Response erklären könnten. Viel Spaß beim Lesen!

Andreas Michalsen, Essen/Berlin

\section{And the Winner Is ...}

\section{Little P, Lewith G, Webley F, Evans M, Beattie A, Middleton K, Barnett J, Ballard K, Oxford F, Smith P, Yardley L, Hollinghurst S, Sharp D: Randomised con- trolled trial of Alexander technique lessons, exercise, and massage (ATEAM) for chronic and recurrent back pain. BMJ 2008;337:a884. doi: 10.1136/bmj.a884.}

Objective: To determine the effectiveness of lessons in the Alexander technique, massage therapy, and advice from a doctor to take exercise (exercise prescription) along with nurse delivered behavioural counselling for patients with chronic or recurrent back pain.

Design: Factorial randomised trial.

Setting: 64 general practices in England.

Participants: 579 patients with chronic or recurrent low back pain; 144 were randomised to normal care, 147 to massage, 144 to six Alexander technique lessons, and 144 to 24 Alexander technique lessons; half of each of these groups were randomised to exercise prescription.

Interventions: Normal care (control), six sessions of massage, six or 24 lessons on the Alexander technique, and prescription for exercise from a doctor with nurse delivered behavioural counselling.

Main Outcome Measures: Roland Morris disability score (number of activities impaired by pain) and number of days in pain.

Results: Exercise and lessons in the Alexander technique, but not massage, remained effective at one year (compared with control Roland disability score 8.1 : massage $-0.58,95 \%$ confidence interval -1.94 to 0.77 , six lessons $-1.40,-2.77$ to $-0.03,24$ lessons $-3.4,-4.76$ to -2.03 , and exercise $-1.29,-2.25$ to -0.34$)$. Exercise after six lessons achieved $72 \%$ of the effect of 24 lessons alone (Roland disability score -2.98 and -4.14 , respectively). Number of days with back pain in the past four weeks was lower after lessons (compared with control median 21 days: 24 lessons -18, six lessons -10 , massage -7 ) and quality of life improved significantly. No significant harms were reported.
Conclusions: One to one lessons in the Alexander technique from registered teachers have long term benefits for patients with chronic back pain. Six lessons followed by exercise prescription were nearly as effective as 24 lessons.

Trial Registration: National Research Register N0028108728.

\section{Kommentar - Andreas Michalsen, Essen/Berlin}

Diese Studie aus dem Hause Lewith ist in dreierlei Hinsicht bemerkenswert. Sie überprüft zunächst die Wirksamkeit eines in Deutschland noch nicht sehr etablierten Therapieverfahrens der Komplementärmedizin. Weiter sind das Design und die Studienmethodik pragmatisch und von höchster Qualität zugleich. Schließlich sind die Ergebnisse eindrucksvoll.

Zum ersten Punkt: Evaluiert wurde die Alexander-Technik. Die Alexander-Technik ist eine Körpertherapie, die nach ihrem Erfinder, dem Australier Frederick M. Alexander (1869-1955) benannt wurde. Alexander war ursprünglich Schauspieler und entsprechend fand die Therapie später auch vielfach unter Schauspielern, Tänzern und Musikern Verbreitung. Die Alexander-Technik versteht sich als Lehr- und Behandlungsmethode zur Veränderung falscher, oder besser: ungünstiger, Bewegungs-, Haltungs- und Verhaltensmuster, die häufig die Ursache für chronische Schmerzen des Bewegungsapparates sind. In einem ersten Schritt wird versucht, den Patienten bisherige Bewegungs- und Gewohnheitsmuster erkennen und spüren zu lassen. Danach werden durch verbale Instruktionen und zeigenden Handkontakt bewusstes Innehalten bzw. «Nichtreagieren» und neue körperliche Bewegungsabläufe vermittelt und geübt. Die Alexander-Technik hat damit

\section{KARGER}

Fax +49761 4520714

Information@Karger.de

www.karger.com (c) 2009 S. Karger GmbH, Freiburg

Accessible online at:

www.karger.com/fok 
auch Parallelen zu anderen somatopädagogischen Verfahren wie z.B. der Feldenkrais-Therapie. Die Alexander-Technik ist vor allem in den USA und England bekannt, während sie sich im deutschsprachigen Raum erst langsam verbreitet. An den Kliniken Essen-Mitte hatten wir zweimal Gelegenheit, mit Alexander-Therapeuten zusammenzuarbeiten, und waren jeweils sehr von den damit erzielbaren Behandlungserfolgen beeindruckt.

Wie haben nun Little et al. die Wirksamkeit der Alexander-Technik evaluiert? In einer wahren Multicenter-Studie (64 Praxen) der Primärversorgung wurden 579 Patienten mit chronischen Rückenschmerzen eingeschlossen. Die Studiendauer war mit 12 Monaten für eine Rückenschmerzstudie erfreulich lang und eröffnete damit auch die Möglichkeit, Aussagen über langfristige Wirkungen und somit die praktische Relevanz der untersuchten Verfahren zu treffen. Die Patienten wurden in 8 Gruppen randomisiert. Sie erhielten eine gewöhnliche allgemeinmedizinische Behandlung (Kontrollgruppe), 6 oder 24 h Alexander-Technik über 6 bzw. 20 Wochen oder 6 klassische Massagen über 6 Wochen. Darüber hinaus erhielt jeweils die Hälfte der Patienten jeder Gruppe additiv ein sportliches Bewegungsprogramm. Damit waren interessante Therapievergleiche möglich. Bewegungstherapie ist Standard aller Leitlinien zum chronischen Rückenschmerz, und auch die klassische Massage zeigt in Metaanalysen signifikante Effekte, allerdings zumeist eher kurzzeitig. Die Studienendpunkte wurden nach 3 und nach 12 Monaten erhoben. Da die Alexander-Technik anstrebt, Bewegungsmuster nachhaltig zu verändern, hypothetisierten die Autoren, dass sie im Gegensatz zu den konventionellen Therapien und zur Massage auch noch nach $1 \mathrm{Jahr}$ signifikante Hauptteffekte zeigen sollte. Das Design der Studie ermöglichte es so nicht nur, die grundsätzliche Wirksamkeit, sondern auch die Nachhaltigkeit des Verfahrens zu überprüfen, und schließlich auch der praktischen Frage nachzugehen, ob eine Kombination mit anderen Therapien additive Effekte zeigt. Die auch ansonsten pragmatische Herangehensweise der Studie betonen die Autoren in der Diskussion: 'As this was a large pragmatic, multipractice, multiteacher, multitherapist study, the results are unlikely to be due to the good work of a small number of enthusiasts.'

Damit kommen wir zu den beeindruckenden Ergebnissen der ATEAM-Studie. Nach 3 Monaten hatten die Massage, das Bewegungsprogramm und beide Alexander-TechnikProgramme die Rückenschmerzen signifikant reduziert. Im Roland Disability Index waren folgende Score-Rückgänge zu verzeichnen: Bewegung -0,9, Massage $-2,0,6 \times$ AlexanderTechnik $-1,7$ und $24 \times$ Alexander-Technik $-2,9$. Noch prägnanter waren die Effekte auf die körperliche Lebensqualität. Hier waren die Score-Anstiege bei Massage 2,6, bei Bewegung 3,0, bei $6 \times$ Alexander-Technik 4,4 und bei $24 \times$ Alexander-Technik erstaunliche 7,5 Punkte.

Noch deutlicher wurden die Ergebnisse nach 1 Jahr: Während die Massage keinen Effekt mehr zeigte, fand sich bei 6 $\times$ Alexander-Technik eine Reduktion des Roland Scores von
$-1,4$, bei Bewegung von $-1,3$ und bei $24 \times$ Alexander-Technik von $-3,4$. Dieser Rückgang des Roland Scores um $>40 \%$ bildete sich auch in einem Rückgang der Arbeitsunfähigkeitszeiten von -18 Tagen ab (>80\%). Die körperliche Lebensqualität stieg um 6 bzw. 11 Score-Punkte in der $6 \times$ bzw. $24 \times$ Alexander-Technik-Gruppe an, hingegen nur um 2 Punkte in den Massage- und Bewegungsgruppen.

In der Einzelgruppenbetrachtung nach $1 \mathrm{Jahr}$ waren die Effekte von intensivierter Alexander-Technik und Bewegung nicht additiv. Hingegen war die Kombination aus $6 \times$ Alexander-Technik und Bewegung deutlich wirksamer als 6 $\times$ Alexander-Technik alleine und kam so fast an den Effekt der intensivierten Alexander-Technik heran. Schließlich war die Alexander-Technik sicher und die Adhärenz mit den Behandlungen gut.

Was bleibt zu kommentieren? Die dokumentierten Effekte der Alexander-Technik, insbesondere die Langzeiteffekte sind von klarer klinischer Relevanz. Die Größe der Effekte übertrifft die der meisten anderen nichtpharmakologischen Verfahren, vielleicht mit Ausnahme des Yoga. Insofern sollte nicht lange gezögert werden, die Alexander-Technik in das Therapiemanagement von chronischem Rückenschmerz mit einzubeziehen, und zwar möglichst früh. Und für die Jury der Academy Awards wäre die Wahl einfach: Alexander-Technik ist der Sieger.

Adresse für Sonderdrucke: Paul S. Little, MD, Community Clinical Sciences Division, Southampton University, Aldermoor Health Centre, Southampton, UK, psl3@soton.ac.uk.

Korrespondenzadresse: Prof. Dr. med. Andreas Michalsen, Kliniken Essen Mitte, Knappschafts-Krankenhaus, Innere V, Am Deimelsberg 34a, 45276 Essen, Deutschland, Tel. +49 201 174-25011, a.michalsen@uni-duisburg-essen.de.

\section{Aus der Ferne heilen - Erwartungs- haltung, Beziehungen und Lösungen}

\section{Walach H, Bosch H, Lewith G, Naumann J, Schwarzer $B$, Falk S, Kohls N, Haraldsson E, Wiesendanger H, Nordmann A, Tomasson H, Prescott P, Bucher HC: Effectiveness of distant healing for patients with chronic fatigue syndrome: a randomised controlled partially blinded trial (EUHEALS). Psychother Psycho- som 2008;77:158-166.}

Background: Distant healing, a form of spiritual healing, is widely used for many conditions but little is known about its effectiveness.

Methods: In order to evaluate distant healing in patients with a stable chronic condition, we randomised 409 patients with chronic fatigue syndrome (CFS) from 14 private practices for environmental medicine in Germany and Austria in a two by two factorial design to immediate versus deferred (waiting for 6 months) distant healing. Half the patients were blinded and half knew their treatment allocation. Patients were treated for 6 months and allocated to groups of 3 healers from a pool of 462 healers in 21 European countries with different healing traditions. Change in 
Mental Health Component Summary (MHCS) score (SF-36) was the primary outcome and Physical Health Component Summary score (PHCS) the secondary outcome.

Results: This trial population had very low quality of life and symptom scores at entry. There were no differences over 6 months in post-treatment MHCS scores between the treated and untreated groups. There was a non-significant outcome $(\mathrm{p}=0.11)$ for healing with PHCS $(1.11 ; 95 \% \mathrm{CI}$ -0.255 to 2.473 at 6 months $)$ and a significant effect $(\mathrm{p}=0.027)$ for blinding; patients who were unblinded became worse during the trial $(-1.544$ $95 \%$ CI -2.913 to -0.176$)$. We found no relevant interaction for blinding among treated patients in MHCS and PHCS. Expectation of treatment and duration of CFS added significantly to the model.

Conclusions: In patients with CFS, distant healing appears to have no statistically significant effect on mental and physical health but the expectation of improvement did improve outcome.

\section{Kommentar - Arndt Büssing, Witten/Herdecke}

Vor einigen Jahren ging ein Aufschrei der Entrüstung durch die Scientific Community, als Byrd im Rahmen einer prospektiven, randomisierten, doppelblinden Studie veröffentlichte, dass Patienten mit koronarer Herzerkrankung signifikant weniger Komplikationen hatten, wenn für sie (außerhalb des Krankenhauses) gebetet wurde [1]. Die Wirkung des fürbittenden Gebetes wurde daraufhin immer wieder untersucht, da von Kritikern als Begründung für die (unerwarteten) positiven Ergebnisse unter anderem methodologische Unzulänglichkeiten angeführt worden waren (die wohl auch bestanden). In den methodisch zumeist besseren Folgestudien wurden die Ergebnisse sowohl bestätigt als auch widerlegt. Ein eindeutiges Urteil war bis vor Kurzem nicht zu fällen. Immer wieder wurde angeführt, dass eine positive Erwartungshaltung der Patienten eine wichtige Rolle für die Interpretation der Ergebnisse spielen würde, diese aber nicht adäquat berücksichtigt sei.

Kompliziert wurde die Sachlage jedoch, als Benson et al. 2006 [2] in einer methodisch gut geplanten Studie zeigten, dass bei Patienten nach einer Bypass-Operation geringfügig, aber signifikant mehr postoperative Komplikationen auftraten, wenn sie wussten, dass für sie gebetet wurde. Hier gab es drei Interventionsgruppen: Diejenigen, denen mitgeteilt wurde, dass möglicherweise für sie gebetet werden würde (und für die dann tatsächlich gebetet wurde oder eben nicht), und diejenigen, die wussten, dass für sie gebetet würde (und für die auch tatsächlich gebetet wurde). Als evidenzorientierter Arzt müsste man nun konsequenterweise seine Patienten dahingehend beraten, dass niemand für sie beten solle, wenn eine Bypass-Operation für sie ansteht. - Das ist so natürlich Unsinn, wirft aber ein interessantes Licht auf den theoretischen Hintergrund vieler Studien in diesem Kontext: Wie soll man sich von einer positiven Erwartungshaltung und/oder einer positiven Zuwendung unabhängige Wirkprozesse vorstellen, wenn man nicht entweder eine Übertragung «subtiler Energien» oder die «Intervention höherer Wesenheiten» voraussetzt, die ab einer gewissen Intensitätsschwelle bestimmter
Heilungsrituale mit Gesundheitsgewährung im Sinne der Studienintervention reagieren?

Ein pragmatischer Ansatz wäre, diese Fragen beiseitezuschieben und einfach zu prüfen, ob es nicht trotzdem funktionieren könnte. Vor einigen hundert Jahren hätte sich auch niemand vorstellen können, dass man in Echtzeit transatlantisch kommunizieren kann. (Wie dies wirklich funktioniert, ist auch nur wenigen klar.)

Genau das haben Walach und Mitarbeiter gemacht, als sie in ihrer aktuellen Untersuchung (EUHEALS) dem Phänomen der Fernheilung nachgingen. Nach Screening wurden 411 Patienten mit CFS in vier unterschiedliche Studienarme randomisiert. Hierzu wurde ein $2 \times 2$ Faktoren-Design gewählt: verblindet / nicht verblindet sowie unmittelbare / verzögerte Intervention. Von den zwei Gruppen, die zur sofortigen Intervention vorgesehen waren, wurde der einen mitgeteilt, dass sie im «Fernheilungs-Arm» seien, der anderen nicht. Die anderen zwei Gruppen wurden dem Warte-Arm zugeordnet; ein Teil von ihnen wurde darüber informiert, dass sie 6 Monate auf eine Behandlung warten müssten, die anderen nicht. Als primäre Outcome-Variable der Studie wurde die mentale gesundheitsbezogene Lebensqualität (SF-36), als sekundäre die physische Gesundheit (SF-36) erfasst.

Die Fernheilenden kamen aus unterschiedlichen Heilungstraditionen und wandten entweder Gebete oder eine «Übertragung heilender Energien», «Licht» oder «Heilkräfte» an. Die Autoren sind bei der Studienplanung sehr pragmatisch vorgegangen, haben (wohl) nicht gefragt, ob und wie eine bestimmte Methode «funktionieren» könnte, und haben daher vorsichtshalber drei Heiler mit ihren jeweiligen Methoden einem Patienten zugewiesen (falls die Fernheilung ineffektiv sein würde, wie es unter anderem begründet wurde). Diese Heiler bekamen den Vornamen des Patienten und ein Bild ausgehändigt sowie die Diagnose mitgeteilt; darüber hinaus mussten sie für jeden Patienten einen wöchentlichen «Heilungsreport» erstellen.

Als aufgeklärter Rationalist mag man sich durch das Studienergebnis in seiner Weltsicht bestätigt fühlen («Wie sollte das auch funktionieren ...?»): Zwischen den behandelten und unbehandelten Patienten gab es nach einer 6-monatigen Interventionsphase hinsichtlich der Prüfziele keine signifikanten Unterschiede. Die Erwartungshaltung der Patienten war in der Studie von Walach und Mitarbeitern hinsichtlich der physischen Gesundheit jedoch die entscheidende Variable: Die Patienten, die wussten, dass sie auf die Intervention warten müssen, zeigten keine Änderung der physischen gesundheitsbezogenen Lebensqualität, während diejenigen, die wussten, dass sie nicht behandelt werden, eine schlechtere Lebensqualität hatten. Hier finden sich Ähnlichkeiten mit der zitierten STEP-Studie von Benson et al. [2], nur dass dort das Wissen um das Fürbittgebet scheinbar mit ungünstigeren Verläufen assoziiert war.

Eine positive Erwartungshaltung und Zuwendung sind in der Placeboforschung als relevante Wirkfaktoren bekannt 
[3]. Hier sind verschiedene endogene Neurotransmitter und/ oder Neurohormone als Mediatoren des sogenannten Placeboeffektes identifiziert worden [4, 5]. Unspezifische Effekte müssen wohl in vielen Bereichen der Medizin angenommen werden, insbesondere bei den besonderen Verfahren der Komplementärmedizin.

Auch wenn die methodisch gut geplante Studie von Walach und Mitarbeitern zur Fernheilung keine positiven Ergebnisse im Sinne der Interventionen gebracht hat, so ist damit doch ein Schritt über den Horizont hinaus angedeutet worden: In der Medizin geht es ebenso wie in der Pflege um eine therapeutische Beziehung, um eine interagierende Begegnung zweier Individuen. Der eine bringt neben Heilungserwartungen, Wünschen und Vorstellungen einen jeweils eigenen biographischen Hintergrund mit, vor dem sich Genesungsprozesse abspielen; der andere bringt sein Fachwissen, persönliche Einstellungen und Präferenzen ein und den Willen, Heilungsprozesse zu initiieren. Wenn man Heilung als einen «Prozess des sich entwickelnden Werdens» auffasst, in dessen Verlauf es zur Auseinandersetzung des Patienten mit den jeweiligen Möglichkeiten und zur Reflexion der Lebensperspektiven kommt [6], dann ist offensichtlich, dass man die Frage nach der Wirksamkeit bestimmter Interventionsformen nicht mehr monokausal betrachten kann. Manche Interventionsformen sind dann vor dem Hintergrund einer positiven Erwartungshaltung und einer therapeutischen Intention sicherlich effektiver als andere, insbesondere wenn hierbei Bewusstwerdungsprozesse beim Patienten als essentielle Moderatoren eines komplexen «Lösungsgeschehens» zum Tragen kommen. - Hierzu ist eine therapeutische Beziehung notwendig. Doch auch das offensichtliche Beziehungsdefizit wurde von einigen Heilern aus der Ferne ganz pragmatisch gelöst: «Fernheilung und Energiearbeit ... Webcam und E-Mail-Beratungsdienste mit Vorkasse». - Wird schon.

\section{Literatur}

1 Byrd RC: Positive therapeutic effects of intercessory prayer in a coronary care unit population. South Med J 1988;81:826-829.

2 Benson H, Dusek JA, Sherwood JB, et al: Study of the Therapeutic Effects of Intercessory Prayer (STEP) in cardiac bypass patients: a multicenter randomized trial of uncertainty and certainty of receiving intercessory prayer. Am Heart J 2006; 151:934-942.

3 Howland RH: Understanding the placebo effect. Part 2: Underlying psychological and neurobiological processes. J Psychosoc Nurs Ment Health Serv 2008; 46:15-18.

4 Haour F: Mechanisms of the placebo effect and of conditioning. Neuroimmunomodulation 2005;12:195-200.

5 Pacheco-López G, Engler H, Niemi MB, Schedlowski M: Expectations and associations that heal: Immunomodulatory placebo effects and its neurobiology. Brain Behav Immun 2006;20:430-446.

6 Büssing A: Kein Weg zurück - Heilung als Prozess des Werdens. Dtsch Z Onkol 2008; 40:28-31.

Adresse für Sonderdrucke: Samueli Institute, European Office, School of Social Sciences, University of Northampton, Northampton, UK. harald. walach@northampton.ac.uk
Kontaktadresse: Prof. Dr. Arndt Büssing, Lehrstuhl für Medizintheorie und Komplementärmedizin, Universität Witten/Herdecke, Gerhard-Kienle-Weg 5, 58313-Herdecke, Tel. +49 2330 623246, ArBuess@yahoo.de.

\section{Entspannungstraining verändert die Genexpression}

\section{Dusek JA, Otu HH, Wohlhueter AL, Bhasin M, Zerbini LF, Joseph MG, Benson H, Libermann TA: Genomic counter-stress changes induced by the relaxation response. PLoS ONE 2008;3(7):e2576.}

Background: Mind-body practices that elicit the relaxation response (RR) have been used worldwide for millennia to prevent and treat disease. The $\mathrm{RR}$ is characterized by decreased oxygen consumption, increased exhaled nitric oxide, and reduced psychological distress. It is believed to be the counterpart of the stress response that exhibits a distinct pattern of physiology and transcriptional profile. We hypothesized that RR elicitation results in characteristic gene expression changes that can be used to measure physiological responses elicited by the RR in an unbiased fashion.

Methods/Principal Findings: We assessed whole blood transcriptional profiles in 19 healthy, long-term practitioners of daily RR practice (group M), 19 healthy controls (group N(1)), and $20 \mathrm{~N}(1)$ individuals who completed 8 weeks of RR training (group N(2)). 2209 genes were differentially expressed in group $M$ relative to group $\mathrm{N}(1)(\mathrm{p}<0.05)$ and 1561 genes in group N(2) compared to group N(1) $(\mathrm{p}<0.05)$. Importantly, 433 $(\mathrm{p}<10(-10))$ of 2209 and 1561 differentially expressed genes were shared among long-term (M) and short-term practitioners (N(2)). Gene ontology and gene set enrichment analyses revealed significant alterations in cellular metabolism, oxidative phosphorylation, generation of reactive oxygen species and response to oxidative stress in long-term and short-term practitioners of daily RR practice that may counteract cellular damage related to chronic psychological stress. A significant number of genes and pathways were confirmed in an independent validation set containing 5 $\mathrm{N}(1)$ controls, $5 \mathrm{~N}(2)$ short-term and $6 \mathrm{M}$ long-term practitioners.

Conclusions/Significance: This study provides the first compelling evidence that the RR elicits specific gene expression changes in short-term and long-term practitioners. Our results suggest consistent and constitutive changes in gene expression resulting from RR may relate to long term physiological effects. Our study may stimulate new investigations into applying transcriptional profiling for accurately measuring RR and stress related responses in multiple disease settings.

\section{Kommentar - Andreas Michalsen Essen/Berlin}

Es ist mehr als 30 Jahre her, dass Herbert Benson in seiner Pionierarbeit die physiologischen Vorgänge während «aktiver Entspannungsphasen» detailliert beschrieb und mit dem Terminus «relaxation response» (RR) belegte. Hierbei wurde zwischen den einzelnen Techniken wenig Unterschied gemacht, es zeigte sich bald, dass die RR durch Meditation, Gebet, Atemtechniken, QiGong oder auch durch Visualisation induziert werden kann. Physiologisch wurde die RR durch eine Absenkung der Herzfrequenz und des Blutdrucks, einen geringeren $\mathrm{O}_{2}$-Verbrauch und eine reduzierte Atemfrequenz charakterisiert. In späteren Arbeiten wurden zudem 
spezifische Muster der Herzfrequenzvariabilität im EKG und spezifische Aktivitäten von kortikalen und subkortikalen Hirnregionen in funktionellen MRTs während der RR dokumentiert. Auf der Suche nach den zugrundeliegenden Mechanismen konnten Hoffmann und Benson [1] in einer frühen Arbeit zeigen, dass regelmäßige RR zu «stress hardiness», also dem geringeren Ansprechen von Zellrezeptoren auf katecholaminerge Stimulation führt. Dies spiegelte damit auch die subjektive Stressreduktion wider, die in klinischen Studien vielfach als herausragende Wirkung von Meditation und weiteren Entspannungstechniken belegt wurde.

Schon länger ermöglichen uns nun die Molekularbiologie und Genetik, bei der Suche nach Wirkmechanismen nicht mehr an den äußeren Zellgrenzen haltzumachen. Im Bereich der Pharmakologie ist es bereits vielfach gelungen, Vorgänge der Signaltransduktion und Genexpression für einzelne Therapien exakt aufzuklären und damit in eine neue Dimension des Nachweises von Wirkmechanismen einer Behandlung zu gelangen.

Vor diesem Hintergund haben Dusek et al. vom BensonHenry Institute für Mind Body Medicine am Massachusetts General Hospital, Boston, eine Meilensteinstudie für die Mind-Body-Medizin und Komplementärmedizin vorgelegt. Das gewählte Studiendesign wurde bereits ähnlich in einigen bildgebenden Studien zur RR eingesetzt. Man vergleicht eine Gruppe von gesunden RR-Langzeitanwendern (zumeist Meditation) zunächst mit einer Kontrollgruppe (RR-«Novizen», Querschnittsstudie). Die Kontrollgruppe erhält dann in einem zweiten Studienteil eine Kurzzeitintervention zum Erlernen und Praktizieren der RR, und die Ergebnisse zum Zeitpunkt 2 können mit dem Ausgangswert und auch mit den Charakteristika der Langzeitanwender verglichen werden.

In den Ergebnissen fanden die Autoren sowohl deutliche Unterschiede in der Genexpression zwischen den Langzeitanwendern und der Kontrollgruppe als auch signifikante Veränderungen in der Kontrollgruppe nach nur 8 Wochen Praktizieren der RR. Die durch die RR induzierten Veränderungen der Genexpression betrafen vor allem Prozesse der oxidativen Phosphorylation und der Bildung von Reactive Oxygen Species (ROS) und der zellulären Antwort auf oxidativen Stress. Damit zeigte sich eine veränderte Genexpression in spezifischen funktionellen Gruppen, gleichsam eine «DownRegulation» von «Stress-Response»-Genen. Dies impliziert, dass durch die RR eine vergrößerte Kapazität für zellulären oxidativen Stress, und damit eine verbesserte Abwehr von Zellschäden entsteht. Besondere Aufmerksamkeit verdient aber vor allem das Ergebnis, dass die Genexpressionsmuster von Langzeit- und Kurzzeitanwendern der RR sehr ähnlich waren. Das heißt, bereits innerhalb weniger Wochen können durch Ausübung eines Entspannungsverfahrens die intrazellulären Mechanismen der vielfach belegten günstigen klinischen Langzeitwirkungen der RR induziert werden.

Die hier publizierten Ergebnisse sind damit auch die Fortschreibung der Arbeit von Bierhaus et al. [2] von der Heidelberger Universitätsklinik. Ihre Arbeitsgruppe konnte belegen und hochrangig publizieren, dass psychosozialer Stress den proinflammatorischen Transkriptionsfaktor NF-kappa B aktiviert, und so erstmals einen Link zwischen Stress und oxidativer Zellschädigung beschreiben. Damit könnte nun der lange währende wissenschaftliche Disput über die Existenz und die Rolle von Stress und Entspannung sein natürliches Ende gefunden haben: Gefühlter Stress ist auch für die Zelle Stress, und der gesundheitsfördernde Effekt von Entspannungsverfahren kann nicht nur mit dem Leuchten von Gehirnarealen assoziiert werden, sondern beginnt nachweislich bereits bei der Genexpression.

\section{Literatur}

1 Hoffmann JW, Benson H, Arns PA, et al: Reduced sympathetic nervous system responsivity associated with the relaxation response. Science 1982;215:190-192.

2 Bierhaus A, Wolf J, Andrassy M, et al: A mechanism converting psychosocial stress into mononuclear cell activation. Proc Natl Acad Sci U S A 2003;100: $1920-5$.

Adresse für Sonderdrucke: Jeffrey Dusek, Ph.D., Benson-Henry Institute for Mind Body Medicine at Massachusetts General Hospital, Chestnut Hill, Boston, MA, USA.

Kontaktadresse: Prof. Dr. med. Andreas Michalsen, Kliniken Essen Mitte, Knappschafts-Krankenhaus, Innere V, Am Deimelsberg 34a, 45276 Essen, Deutschland, Tel. +49 201 174-25011, a.michalsen@ uni-duisburg-essen.de. 